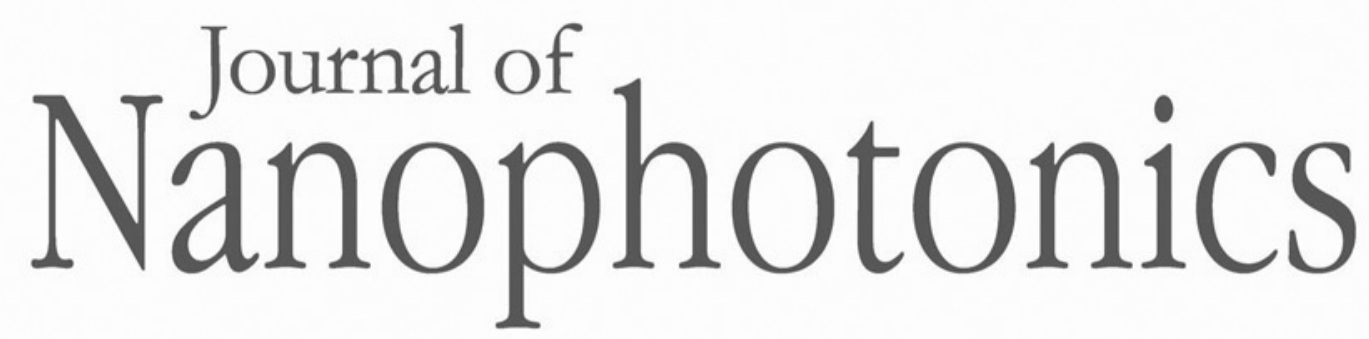

\title{
Ultraluminescent gold core-shell nanoparticles applied to individual bacterial detection based on metal- enhanced fluorescence nanoimaging
}

Daniela Gontero

Alicia V. Veglia

Denis Boudreau

Angel Guillermo Bracamonte 


\title{
Ultraluminescent gold core-shell nanoparticles applied to individual bacterial detection based on metal-enhanced fluorescence nanoimaging
}

\author{
Daniela Gontero, ${ }^{a}$ Alicia V. Veglia, ${ }^{b}$ Denis Boudreau, ${ }^{c}$ and \\ Angel Guillermo Bracamonte ${ }^{\mathrm{b}, *}$ \\ aLaboratorio de Análisis Clínicos y Bacteriológicos, Clínica de la Familia II. Río Tercero, \\ Córdoba, Argentina \\ ${ }^{\text {b} U n i v e r s i d a d ~ N a c i o n a l ~ d e ~ C o ́ r d o b a, ~ I n s t i t u t o ~ d e ~ I n v e s t i g a c i o n e s ~ e n ~ F i ́ s i c o ~ Q u i ́ m i c a ~ d e ~ C o ́ r d o b a ~}$ \\ (INFIQC), Departamento de Química Orgánica, Facultad de Ciencias Químicas, Ciudad \\ Universitaria, Córdoba, Argentina \\ 'Université Laval, Centre d'Optique, Photonique et Laser, Département de Chimie, Québec, \\ Canada
}

\begin{abstract}
Gold core-shell nanoparticles were synthesized based on metallic cores, variable silica shell spacers covered with modified fluorescent silica layers. Ultraluminescent properties were obtained based on metal-enhanced fluorescence (MEF). Different silica spacers were synthesized to optimize the $\mathrm{MEF}$ enhancement factor $\left(\mathrm{MEF}_{\mathrm{EF}}\right)$. An optimal $\mathrm{MEF}_{\mathrm{EF}}$ was determined equal to 9.5 for shorter silica spacers $\left(d_{-\mathrm{SiO}_{2-}}=10 \mathrm{~nm}\right)$. These nanoparticles were deposed on Escherichia coli bacteria at different concentration levels for Bioimaging generation over their surfaces. The best luminescent nanoparticles were deposed on intermediate and higher bacteria concentrations. In the presence of intermediate bacteria concentrations, the ultraluminescent nanoparticles adsorbed showed an increase of 35\% to $45 \%$ compared with individual nanoparticles. To modify the surface of individual bacteria, diluted samples of bacteria were used in which a $20 \%$ decrease in fluorescence emission was measured. In the presence of higher bacteria concentrations, fewer clear and bright images were obtained. At diluted ultraluminescent nanoparticle concentrations, a decrease in brightness and image detail was observed; and in the absence of nanoparticle deposition, no image was recorded. Accordingly, these ultraluminescent gold core-shell nanoparticles have been shown to be useful as platforms for biodetection and tracking applications. (C) 2017 Society of Photo-Optical Instrumentation Engineers (SPIE) [DOI: 10 .1117/1.JNP.12.012505]
\end{abstract}

Keywords: gold core-shell nanoparticles; metal-enhanced fluorescence; bacterial detection; nanoimaging.

Paper 17017SSP received Feb. 26, 2017; accepted for publication Jun. 7, 2017; published online Jun. 28, 2017.

\section{Introduction}

Essential needs related to the biodetection of bacteria in many areas, such as clinical diagnostics, biochemistry, food, environment, and soil and water quality, remain unfulfilled. Within all bacteria known, normally determined by conventional methods, Escherichia coli bacteria require special attention. ${ }^{1}$

Interest in bacterial detection focuses not only on clinical diagnostics but also on the genotyping of new bacteria. From this point of view, new nanosensors integrated in microdevice developments are of high impact for social needs. Cases in point include microdevices for bacterial detection integration, such as a magneto-DNA nanoparticle system for rapid detection and phenotyping of bacteria by polymerase chain reaction, ${ }^{2}$ developed by Weissleder et al. (2013).

*Address all correspondence to: Angel Guillermo Bracamonte, E-mail: gbracamonte@ fcq.unc.edu.ar

$1934-2608 / 2017 / \$ 25.00$ (C) 2017 SPIE

Journal of Nanophotonics

012505-1

Jan-Mar 2018 • Vol. 12(1) 
A further case is a smartphone application for bacterial detection, developed by Wei et al. (2013) from studies based on luminescent nanostructures and fluorescence microscopy developments. ${ }^{3}$ In both cases, magnetic and luminescent nanostructures were used; yet it should be mentioned that not many developments with fluorescent nanoparticles have been reported. However, most are related to magnetic nanoparticles that, due to their intrinsic properties, can be extracted via an electromagnetic field. ${ }^{4}$ For this reason, in the literature developments of magnetic nanoparticles for bacterial separation and detection by additional properties incorporated in the nanostructure, such as fluorophores ${ }^{5}$ and other optical reporters, can be found. ${ }^{6}$

Developments of luminescent nanostructures, such as quantum dots (Qdots) for bacteria detection, were based on their intrinsic luminescent properties, ${ }^{7}$ in addition to being coupled to energy transfer processes, such as Forster reaction electron transfer (FRET) ${ }^{8}$ Interesting results from comparisons of Qdots versus fluorescent labeling with organic fluorophores ${ }^{9}$ can be found in the literature.

But from the viewpoint of signal transduction and energy transfer, the need for design and synthesis of better luminescent nanoparticles as small and stable enhanced emitters exists, and it should be accompanied with additional properties depending of the application as for example well dispersibility and photostability in aqueous media for biophotonics applications. Moreover, easy chemical surface modification and tunable properties open technological applications as nanophotonic systems.

Therefore, the development of new ultraluminescent nanostructures based on plasmonic effects, such as metal-enhanced fluorescence $(\mathrm{MEF})^{10}$ is needed since, as far as we know, there is no report in the field for individual bacteria detection and tracking by nanoimaging. However based on a plasmonic effects, it could be mentioned developments based on $\mathrm{SPR}^{11}$ and SERS, ${ }^{12}$ a highly sensitive technique that from the practical point of view, could be used in flow methods. ${ }^{13}$

Metallic core-shell nanoparticles are versatile platforms that can be used for ultraluminescent nanoparticle design based on MEF due to their stability in aqueous media, relatively easily chemical surface functionalization, decrease in photobleaching of fluorophores attached, and enhanced luminescent properties. Boudreau et al. ${ }^{14}$ developed ultraluminescent silver coreshell nanoparticles for DNA detection based on MEF coupled to FRET with a flow method and nanoimaging system. Moreover, recent studies of silver core-shell nanoparticles and their principal parameters to be controlled showed ultraluminescent properties with many fluorophores, thus opening up future application areas. ${ }^{15}$

Recently, ultraluminescent gold core-shell nanoparticles were reported by Gontero et al. ${ }^{16}$ with no previous reports of these nanoparticles for biosensing existing to the best of our knowledge. These new ultraluminescent gold core-shell nanoparticles are of high interest in nanotechnological applications, as in clinical chemistry, due to bacterial resistance to antibiotics over the last years, bacteria phenotyping, and long time-consuming analytical clinical procedures. Accordingly, this has encouraged us to study further the luminescent properties of gold core-shell nanoparticles as platforms applied to biodetection based on nanoimaging analysis.

The specific objective of this research work was to develop and optimize ultraluminescent gold core-shell nanoparticles for bacteria detection, based on the study of the interaction and luminescent properties of the nanoparticles deposed on $E$. coli bacteria by nanoimaging generation with Laser Fluorescence Microscopy.

\section{Experimental}

\subsection{Apparatus}

UV-vis and spectrofluorimetric determinations were carried out in a Varian UV-50 Carry 50 Conc. and a Cary Eclipse, respectively. Lifetime measurements were done with a PicoQuant, FluoTime 2000.

An Olympus Fluoview FV1000 confocal laser scanning microscope was used for fluorescence microscopy images and for bright-field confocal microscopy.

Delsa $^{\mathrm{TM}}$ nano submicron particle size-zeta potential particle analyzer and transmission electron microscopy (TEM), TEM JEM-1230, JEOL, with an operating voltage of $200 \mathrm{kV}$, were used for determination of nanoparticle size. 
An ultrasonic bath (Branson 2510) was used for the dispersion of the reagents and colloidal dispersions. The centrifugation was done using an Eppendorf Centrifuge 5804 (range 7500 to $8000 \mathrm{rpm})$.

Data analysis was performed with origin (scientific graph system), version 8 .

\subsection{Reagents}

Water was obtained using a millipore apparatus. RhB (99\% purity, Sigma-Aldrich), hydrogen tetrachloroaurate, $\mathrm{HAuCl}_{4} 3 \mathrm{H}_{2} \mathrm{O}$ (99\%, Sigma-Aldrich), citrate sodium tribasic dehydrate (99\%, ACS reagent, Sigma-Aldrich), poly(vinyl)pyrrolidone 40 (PVP 40, Sigma-Aldrich), tetraethyl orthosilicate (TEOS, 98\%, Sigma-Aldrich), ethanol (Sintorgan, high liquid performance cromatography grade), 3-(aminopropyl)triethoxysilane [3(aminopropyl)triethoxysilane (APS), 98\%, Sigma-Aldrich], $N$-hydroxysuccinimide (NHS), and $N$-(3-dimethylaminopropyl)$N^{\prime}$-ethylcarbodiimide hydrochloride [N-(3-dimethylaminopropyl)-N'- ethylcarbodiimide hydrochloride (EDC), 98\%, Sigma-Aldrich]; sodium cyanide (95\%, Sigma-Aldrich).

E. coli as gram negative bacteria were purchased from Sigma-Aldrich. The components of the culture medium for bacteria growth used in growing and maintaining bacterial cultures were supplied by the same company.

\subsection{General Procedure}

The gold nanoparticles were synthesized by the citrate reduction method ${ }^{17}$ of $\mathrm{HAuCl}_{4}$ and were afterward covered with PVP 40. The resulting nanoparticles were then redispersed in anhydrous ethanol [mother solution, $(\mathrm{Au} \mathrm{NPs})=3.88 \times 10^{10} \mathrm{NPs} / \mathrm{ml}$, diameter $=41.5 \mathrm{~nm}$ ]. After that the surface of the nanoparticles was modified with variable silica spacer lengths obtained by the classical Stober method. ${ }^{18}$ For a typical synthesis of gold core-shell nanoparticles $\left(\mathrm{Au} @ \mathrm{SiO}_{2}\right)$, 9 to 10 -nm silica shell $7.5 \mu \mathrm{l}$ of TEOS $10 \%$ (at $\mathrm{pH}=8$ to 9 by addition of $\mathrm{NH}_{4} \mathrm{OH}$ ) was added vigorously to $4 \mathrm{ml}$ of PVP-covered gold nanoparticles.

Then it was covalently bonded to RhB with APS by NHS/EDC activation to afford an RhBAPS-conjugated solution. For RhB linking over the silica surface, increasingly variable volumes of this solution were added to $1 \mathrm{ml}$ of $\mathrm{Au} @ \mathrm{SiO}_{2}$ with continuous stirring.

The reaction time was $20 \mathrm{~min}$ and immediately after a second, thin silica shell was added via a solution of TEOS $2.5 \%$. The reaction was allowed to react for $24 \mathrm{~h}$.

For the $\mathrm{MEF}_{\mathrm{EF}}$, coreless silica nanoparticles $\left[(-) @ \mathrm{SiO}_{2}-\mathrm{RhB}\right]$ were obtained using the sodium cyanide leakage method ${ }^{19}$ (Vortex overnight of samples was applied in the presence of sodium cyanide to completely digest the metallic core). The $\mathrm{MEF}_{\mathrm{EF}}=\left[\right.$ intensity of $\mathrm{AU} @ \mathrm{SiO}_{2}-$ $\mathrm{RhB} /$ intensity of (-) @ $\left.\mathrm{SiO}_{2}-\mathrm{RhB}\right]$.

At each step in the synthesis, the nanoparticles were centrifuged and redispersed in anhydrous ethanol. Centrifugation was done between 7400 and $8000 \mathrm{rpm}$ depending on the sample. Lower centrifugation speed was applied for $(-) @ \mathrm{SiO}_{2}-\mathrm{RhB}$ and $\mathrm{AU} @ \mathrm{SiO}_{2}-\mathrm{RhB}$ to avoid $\mathrm{RhB}$ leakage (see synthesis steps of $\mathrm{AU} @ \mathrm{SiO}_{2}-\mathrm{RhB}$ in Fig. 1).

Fluorescence emission spectra were measured with an excitation wavelength equal to the wavelength of maximum absorption. To confirm that the maximal emission fluorescence was measured under these conditions, the excitation wavelength was evaluated by measuring the 3-D fluorescence emission spectrum.

For emission and excitation fluorescence spectra, the excitation and emission bandwidths were set at 5 and $10 \mathrm{~nm}$, respectively. The PMT gain was medium. All the measurements were performed at $25.0^{\circ} \mathrm{C} \pm 0.1^{\circ} \mathrm{C}$, with the temperature of the cell compartment being controlled with a Haake K10 circulator under continuous stirring. The 3-D fluorescence experiments were done by varying the excitation and emission wavelengths in the range of 520 to $570 \mathrm{~nm}$ and 550 to $650 \mathrm{~nm}$, respectively. The excitation wavelength chosen was an intermediate value between the plasmon of the gold nanoparticle and the gold core-shell nanoparticle with a 9- to 10-nm silica spacer of $542.0 \mathrm{~nm}$ for a better RhB excitation.

The lifetime measurements of $\mathrm{AU} @ \mathrm{SiO}_{2}-\mathrm{RhB}, \mathrm{AU} @ \mathrm{SiO}_{2}$, and $\mathrm{RhB}$ free were performed in ethanol. In all measurements, low concentrations $(\sim 3.88108 \mathrm{NPs} / \mathrm{ml}$ that corresponds to the concentrated colloidal dispersion or a dilution factor of 100 of the initial gold mother solution) of gold nanoparticles were used. 

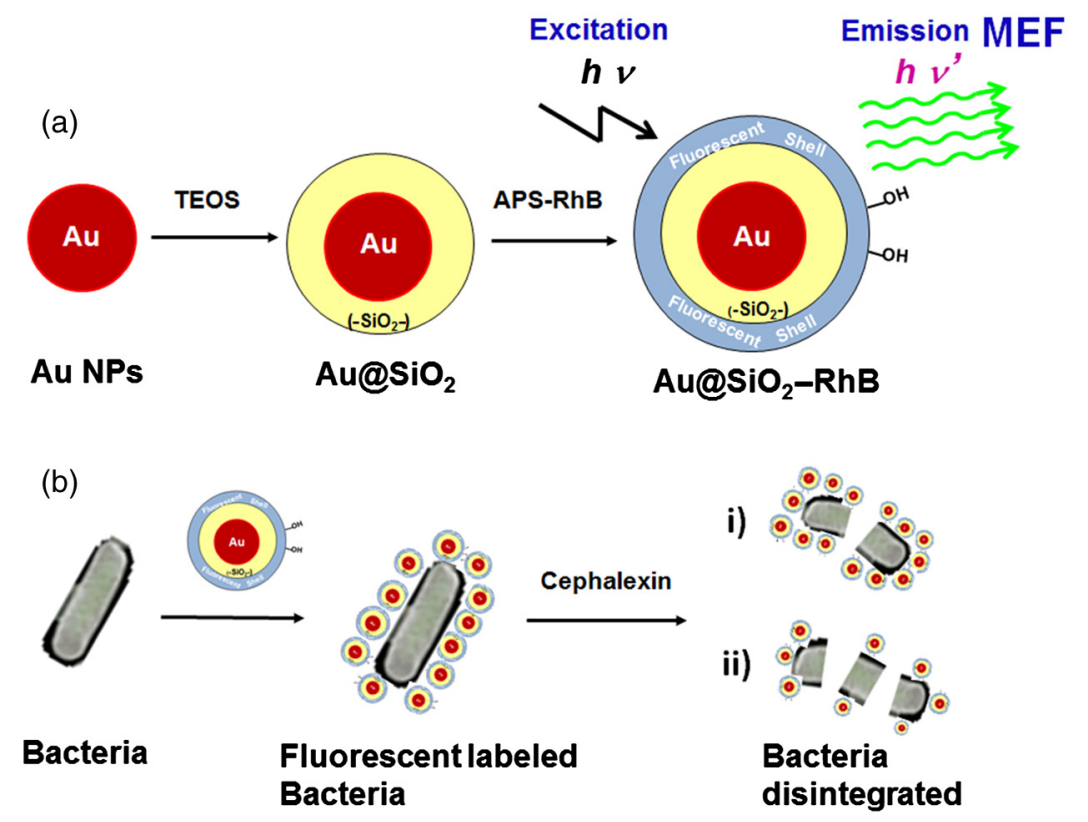

Fig. 1 Scheme of (a) $\mathrm{AU} @ \mathrm{SiO}_{2}-\mathrm{RhB}$ synthesis (b) bacterial modification, (i) concentrated and (ii) diluted ultraluminescent labeling.

For bacteria-nanoparticle interaction, a dispersion of bacteria prepared from the colonies obtained from the culture growth media was prepared. Growth rates and bacterial concentrations were determined by measuring optical density (OD) at $600 \mathrm{~nm}$ every $30 \mathrm{~min}$ (OD of 0.1 corresponds to a concentration of $10^{8}$ cells $/ \mathrm{ml}$ ). From a concentrated dispersion of bacteria in aqueous media, dilutions were prepared to observe on the bright-field confocal microscope individual bacteria to microaggregates of bacteria. For bacterial fluorescent labeling, the dispersions prepared were in contact with ultraluminescent $\mathrm{AU} @ \mathrm{SiO}_{2}-\mathrm{RhB}$ nanoparticles from 0.9 to $5 \times 10^{8} \mathrm{NPs} / \mathrm{ml}$ for $1 \mathrm{~h}$. After that samples were observed by fluorescence microscopy with a minimal volume, adding one drop $(50 \mu \mathrm{l})$ over a microscope glass slide (covered after addition with a cover glass).

\section{Results and Discussion}

\subsection{Characterization of Ultraluminiscent $A U @ S O_{2}-R h B$ Nanoparticles}

To optimize the luminescent properties, gold core-shell nanoparticles with variable shell thickness, between 6 and $14 \mathrm{~nm}$, were synthesized. Core dimension was 40.0-nm diameter to obtain an optimal plasmonic and fluorophore interaction (Fig. 1). The RhB overlapped very well with this nanoparticle diameter $\left(\lambda_{\text {max.Abs. }}=537.0\right.$ for gold nanoparticles, and $\lambda_{\text {max.Abs. }}=549.0$ for $\mathrm{RhB})$. We observed a shift to longer wavelengths in the presence of the silica spacer of $9 \mathrm{~nm}$ to 545.0 to $546.0 \mathrm{~nm}$ [see Fig. 2(a)].

Gold core-shell nanoparticles were grafted with a fluorescent silica layer at each silica spacer obtained 6 to $7 \mathrm{~nm}$ and 9 to $10 \mathrm{~nm}$ as previously carried out in our laboratory. In the presence of the optimal concentration load of RhB determined $0.375 \mu \mathrm{M}$ and the silica spacer length of 7 to $9 \mathrm{~nm}, 3$-D fluorescence spectra were measured [see Fig. 2(b)]. It was shown that for $\lambda_{\text {exc. from }}$ 337.0 to $350.0 \mathrm{~nm}$, the maximal emission fluorescence was measured for $\mathrm{AU} @ \mathrm{SiO}_{2}-\mathrm{RhB}$ with a silica shell of $9 \mathrm{~nm}$.

To evaluate the presence of the core on the emission fluorescence, leaking of $\mathrm{AU} @ \mathrm{SiO}_{2}-$ $\mathrm{RhB}$ was done by applying the sodium cyanide method, and the $\mathrm{MEF}_{\mathrm{EF}}$ determined was close to 10 by improving $\lambda_{\text {exc. }}$ and the silica spacer length (see Fig. 3). An intermediate $\lambda_{\text {exc. }}$ between $\mathrm{Au}$ and $\mathrm{AU} @ \mathrm{SiO}_{2}$ plasmon was applied for an optimal Au plasmon and $\mathrm{RhB}$ excitation in the near and far fields, respectively. 

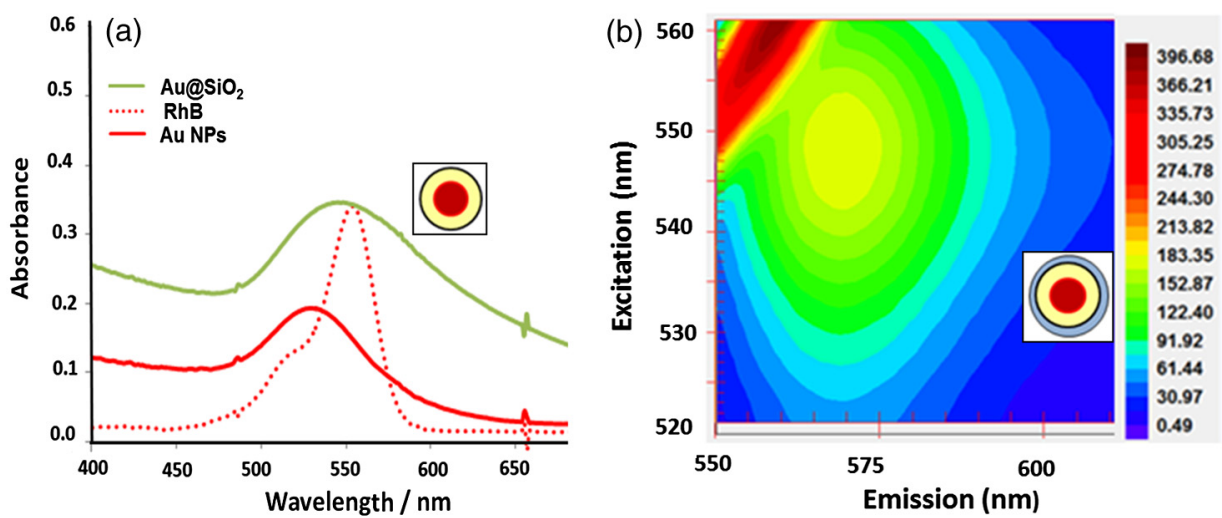

Fig. 2 (a) UV absorption spectra of Rhodamine $\mathrm{B}$, gold and $\mathrm{AU} @ \mathrm{SiO}_{2}$ nanoparticles dispersed in ethanol/water media. The box inset represents the synthesis scheme of fluorescent gold coreshell nanoparticles. (b) 3-D fluorescence spectra with $\Delta \lambda_{\mathrm{exc}}=1.0 \mathrm{~nm}$ and emission from 550 to $700 \mathrm{~nm}$.

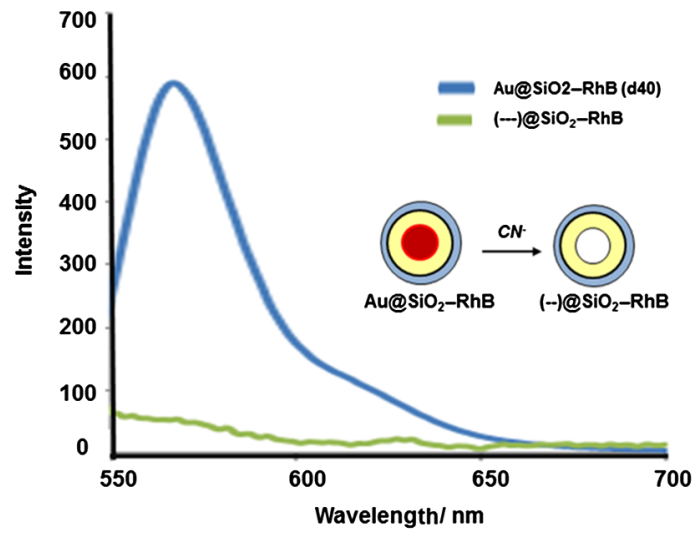

Fig. 3 Effect of gold core on RhB emission fluorescence for $\mathrm{AU} @ \mathrm{SiO}_{2}-\mathrm{RhB}$ nanoparticles with silica spacer $=9 \mathrm{~nm}$ and $[\mathrm{RhB}]=0.073 \mu \mathrm{M}$.

The $\mathrm{MEF}_{\mathrm{EF}}$ (Fig. 4) showed distance dependence, with maximum values of 9.9 for 9- to 10-nm, 8.0 for 6- to 7-nm, and 7.4 for 12- to 14-nm silica spacer lengths determined. At longer distances, 25.0 to $30.0 \mathrm{~nm}$, a sharp diminution was observed. Both effects can be attributed to the interaction of the electromagnetic field in the near field of the gold surface nanoparticle and the $\mathrm{RhB}$. At the right distance for an optimal interaction of the electromagnetic field in the near field

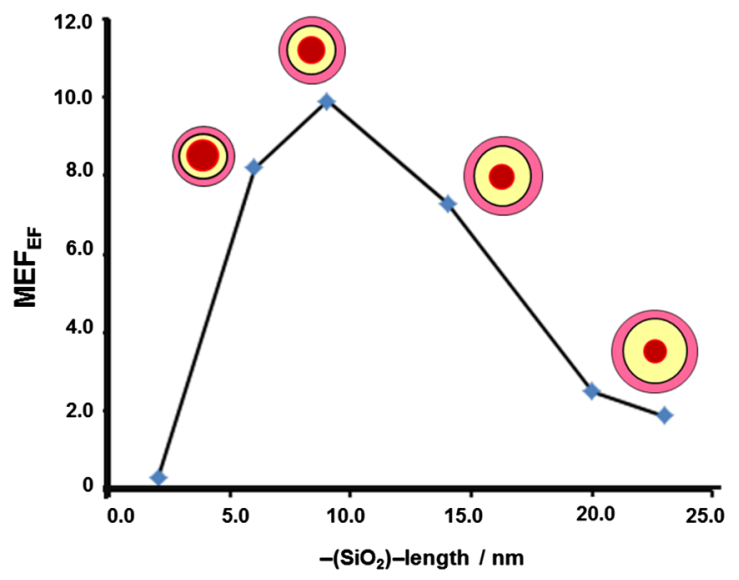

Fig. 4 Effect of the silica spacer length on MEF enhancement factor of $A U @ \mathrm{SiO}_{2}-\mathrm{RhB}$. 


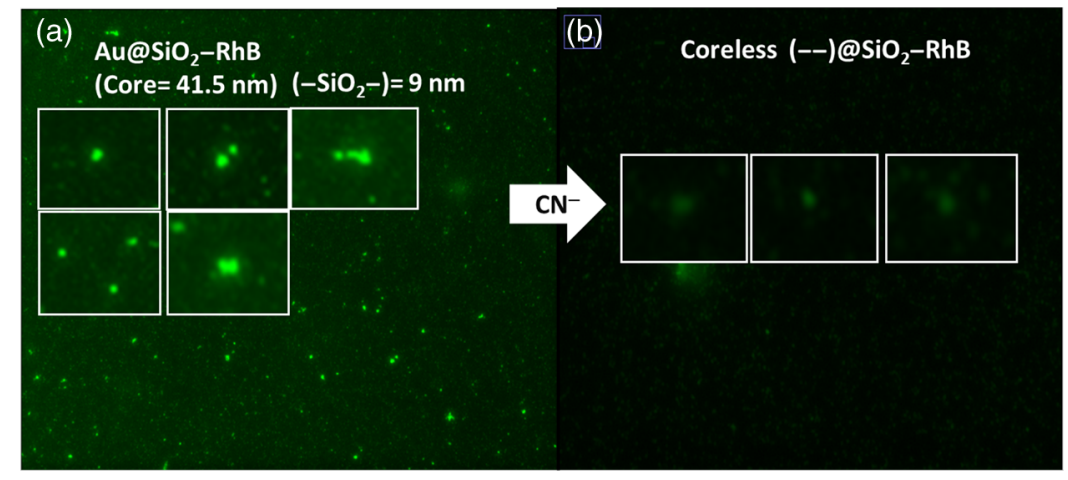

Fig. 5 Fluorescent microscopy of (a) $\mathrm{AU} @ \mathrm{SiO}_{2}-\mathrm{RhB}$, silica spacer 9 to $10 \mathrm{~nm}$. (b) Coreless nanoparticles (-)@ $\mathrm{SiO}_{2}-\mathrm{RhB}$ obtained after sodium cyanide addition. The insets are amplification of luminescent dimers, trimers, and individual core-shell and coreless nanoparticles. $[\mathrm{RhB}]=0.073 \mu \mathrm{M}$.

of the nanoparticle surface and fluorophore, an increase in absorption was produced at the maximal absorption wavelength of the fluorophores, and by this manner, an enhanced emission was produced. $^{20}$

Clear and bright ultraluminescent $\mathrm{AU} @ \mathrm{SiO}_{2}-\mathrm{RhB}$ nanoparticles were obtained compared with coreless nanoparticles by fluorescence microscopy (see Fig. 5). These core-shell nanoparticles were described as photostable, decreasing photobleaching; they were shown to be stable in aqueous media with a particularly good dispersibility and biocompatibility especially in the case of gold core-shell nanoparticles. In all cases, the $\mathrm{MEF}_{\mathrm{EF}}$ obtained from nanoimaging reached values higher than 10, which can rise close to 30 to 40 . For these reasons, these nanoparticles were applied to fluorescent labeling for E. coli bacteria detection to develop a bioanalytic method for diagnostics based on nanoimaging, as was recently reported in our laboratory with an $\mathrm{MEF}_{\mathrm{EF}}$ close to 7 to $8 .{ }^{16}$

\subsection{Deposition of $\mathrm{AU} @ \mathrm{SiO}_{2}-\mathrm{RhB}$ Nanoparticles on Bacteria}

Deposition of AU@ $\mathrm{SiO}_{2}-\mathrm{RhB}$ nanoparticles by simple noncovalent interaction was observed by fluorescence microscopy. Clear images of bacteria were generated with two distributions of emission intensities over the bacteria [see Fig. 6(a)]. Bright-field confocal microscopy showed fewer details in comparison with fluorescence microscopy [see Fig. 6(b)]. Individual and aggregated bacteria were obtained. On the luminescent hot spots from the bacteria labeled in the aggregated state, it was possible to discriminate the bacteria in the bulk; yet, with the control of bright-field confocal microscopy, it was not possible to achieve similar details. In the absence of $\mathrm{AU} @ \mathrm{SiO}_{2}-\mathrm{RhB}$ nanoparticles, no image of bacteria was obtained [see Fig. 6(c)]. However, control by bright-field confocal microscopy was positive [see Fig. 6(d)]. Therefore, it was possible to get fluorescent labeled bacteria with an improved level of detail as compared with brightfield confocal microscopy.

\subsection{Characterization of Bacteria Labeled with $\mathrm{AU} @ \mathrm{SiO}_{2}-R h B$ Nanoparticles}

Measurements by dynamic light scattering (DLS) showed a bacterial control dimension of around $1500 \mathrm{~nm}$ and higher dimension (only one large size distribution); in the presence of $\mathrm{AU} @ \mathrm{SiO}_{2}-\mathrm{RhB}$ nanoparticles, it was possible to measure a higher distribution for bacteria (6000 to $7000 \mathrm{~nm}$ ) derived from (3 to 4) bacterial aggregates and a second smaller distribution of 100 to $150 \mathrm{~nm}$ from the nanoaggregates of nanoparticles (dimmers and trimers). The higher distribution corresponds to aggregation of modified bacteria that corresponds to the sum of the bacterial control dimension and the nanoparticle shell of 100 to $150 \mathrm{~nm}$. When the bacteria were modified with $\mathrm{AU} @ \mathrm{SiO}_{2}-\mathrm{RhB}$ nanoparticles, a thinner Gaussian distribution was observed as compared with the nonlabeled one, which can be attributed to an increase in the dispersibility of microstructures. 


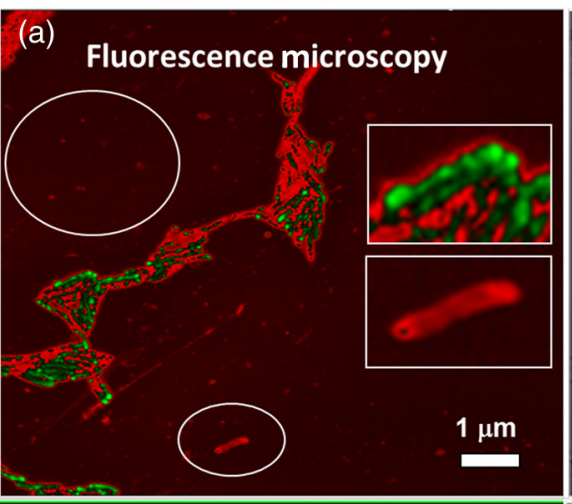

(c) Fluorescence microscopy

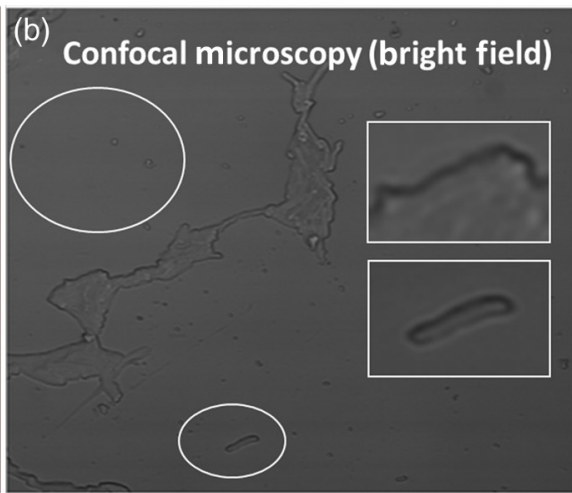

(d) Confocal microscopy (bright field)

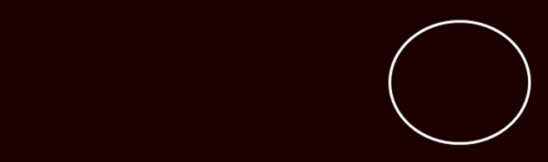

Fig. 6 (a) Fluorescence microscopy of $E$. coli bacteria labeled with luminescent gold core-shell nanoparticles. (b) Bright-field confocal microscopy of the same fluorescent labeled bacteria spot shown in (a). (c) Fluorescent microscopy of E. coli bacteria nonlabeled. (d) Bright-field confocal microscopy of the same bacteria nonlabeled spot shown in (c). Edition of fluorescent microscopy image carried out by two-color analysis with red-green. The green hot spots show higher luminescence intensity than the red ones. The insets are amplification of luminescent bacteria labeled; and white circles indicate luminescent nanoparticles and luminescent bacteria labeled.

This distribution of dimension of fluorescent labeled bacteria in the presence of $\mathrm{AU} @ \mathrm{SiO}_{2}-$ $\mathrm{RhB}$ nanoparticles was modified drastically, with the addition of antibiotic Cephalexin (see Fig. 7). Fragments of bacteria of 390 and $700 \mathrm{~nm}$ for diluted and concentrated nanoparticle conditions were measured, respectively. Hence, it was possible to observe a protective effect

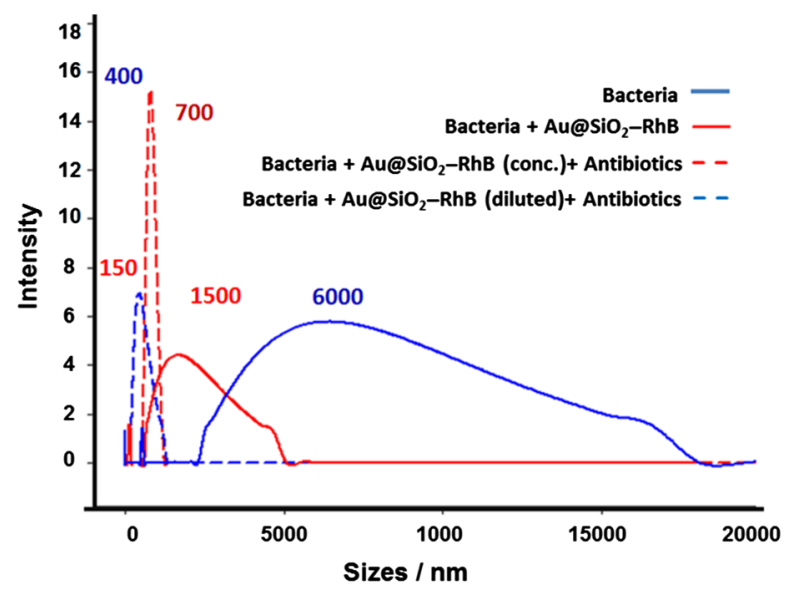

Fig. 7 DLS of E. coli bacteria labeled and nonlabeled with ultraluminescent gold coreshell nanoparticles and labeled bacteria in the presence of Cephalexin antibiotic. Concentration of nanoparticles $=5.0 \times 10^{8} \mathrm{NPs} / \mathrm{ml}$ for concentrated and $0.9 \times 10^{8} \mathrm{NPs} / \mathrm{ml}$ for diluted conditions. 


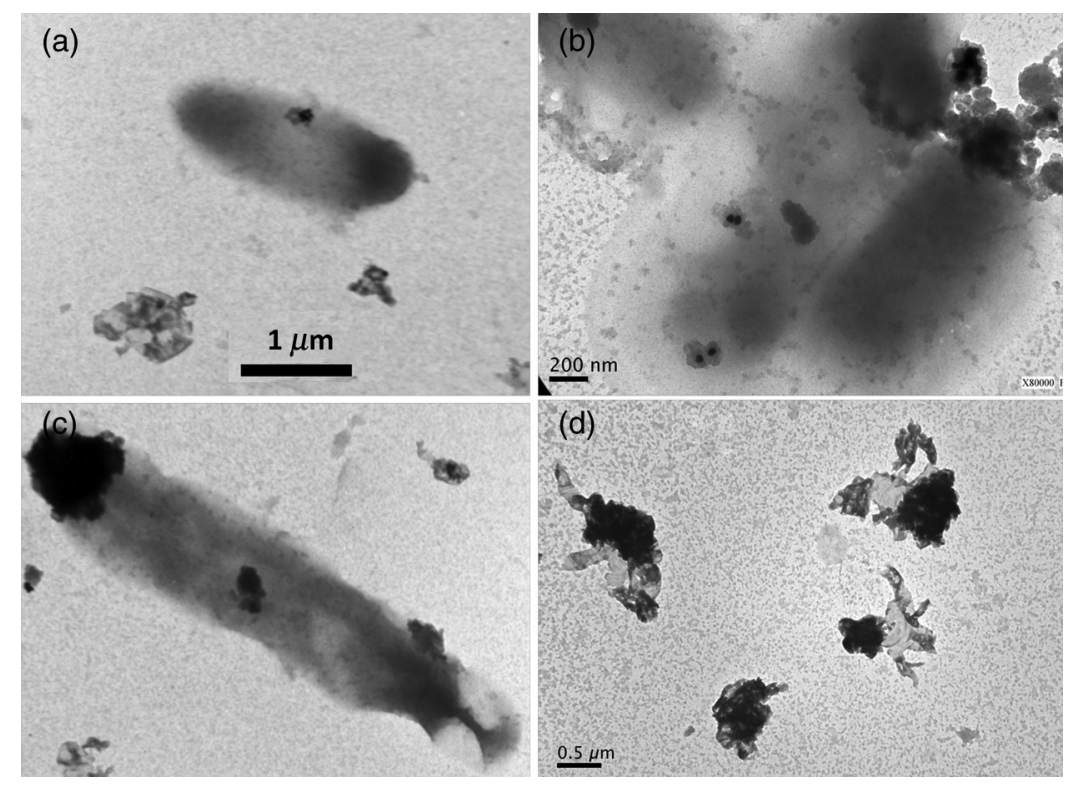

Fig. 8 TEM images of bacteria modified with $\mathrm{AU} @ \mathrm{SiO}_{2}-\mathrm{RhB}$. (a) Typical 1500-nm bacteria fluorescent labeled with low concentration of nanoparticles. (b) Aggregate of concentrated fluorescent labeled bacteria. (c) 2300-nm distribution of fluorescent labeled bacteria. (d) Disintegrated concentrated fluorescent labeled bacteria in the presence of Cephalexin.

by varying the $\mathrm{AU} @ \mathrm{SiO}_{2}-\mathrm{RhB}$ nanoparticles' concentration, which can be explained by the adsorption of the nanoparticles over $E$. coli bacteria.

By TEM, $1500 \mathrm{~nm}$ and higher bacteria were measured as found in DLS. Moreover, deposition of individual nanoparticles was found over all the surface of the bacteria [see Fig. 8(a)] and dimmers, trimmers, and nanoaggregates were also found trapped on the extremity of one side of the bacteria [see Figs. 8(b) and 8(c)]. This observation was related to an asymmetrical labeling of the bacteria correlates with the intensity analysis of fluorescence images, where higher intensity was observed at one end of the bacterium.

In the presence of Cephalexin, it was possible to observe the bacteria disintegrated [see Fig. 8(d)].

\subsection{Effect of Bacterial Concentration on Imaging Quality}

The effect of bacterial concentration on Imaging could be observed by varying the bacterial concentration for $\mathrm{AU} @ \mathrm{SiO}_{2}-\mathrm{RhB}$ nanoparticles' interaction. We compared the intensity of the fluorescent labeled bacteria with the highest luminescence intensity of individual luminescent nanoparticles ( $100 \%$ of intensity). A decrease in intensity of $20 \%$ was observed for individual bacteria and $35 \%$ to $40 \%$ for higher bacteria concentration; however, in the presence of an intermediate bacterial concentration, an increase of $40 \%$ was measured (see Fig. 9). For individual bacteria and intermediate concentration of bacteria, clear and well-defined microstructures were found; but in the presence of the highest bacterial concentration, less clear microaggregates with lower resolution were measured.

With the analysis of individual bacteria edited by two colors (red and green), improved images, with more detail, were obtained compared with only a green color [see Figs. 10(a) and 10(b)]. The two color edition showed two distributions of emission intensities.

Three distributions of luminescent nanoparticles were observed: the clear and strong luminescent nanoparticles (100\%, green color), a weaker distribution (50\%, red color), and one with a strong green luminescent core surrounded with a weaker red emission (core with $100 \%$ and a shell with 40 $50 \%$ ). The most intense luminescent nanoparticles were individual hot luminescent dots (100\%).

Moreover, measured differences of intensity percentages over the bacterium surface are described as follows: $80 \%$ of intensity at the end of the bacterium and $40 \%$ surrounding it compared with the most intense luminescent nanoparticles (100\%). 


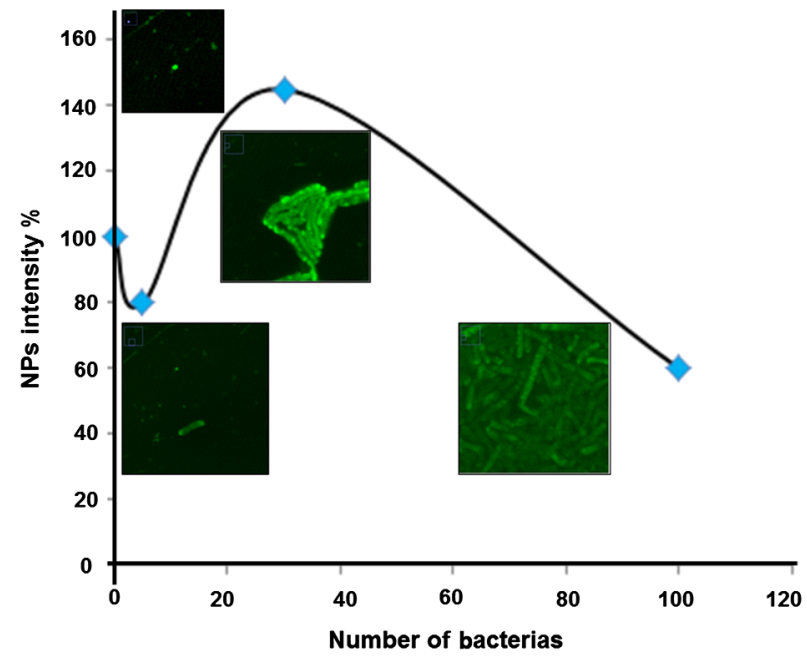

Fig. 9 Intensity percentage of luminescent gold core-shell nanoparticles, $\mathrm{AU} @ \mathrm{SiO}_{2}-\mathrm{RhB}(\mathrm{NPs}$ intensity percentage) versus number of bacteria. Hundred nanoparticle intensity percentage corresponds to individual luminescent gold core-shell nanoparticles. The graph insets represent hot spots analyzed at different bacterial concentrations.
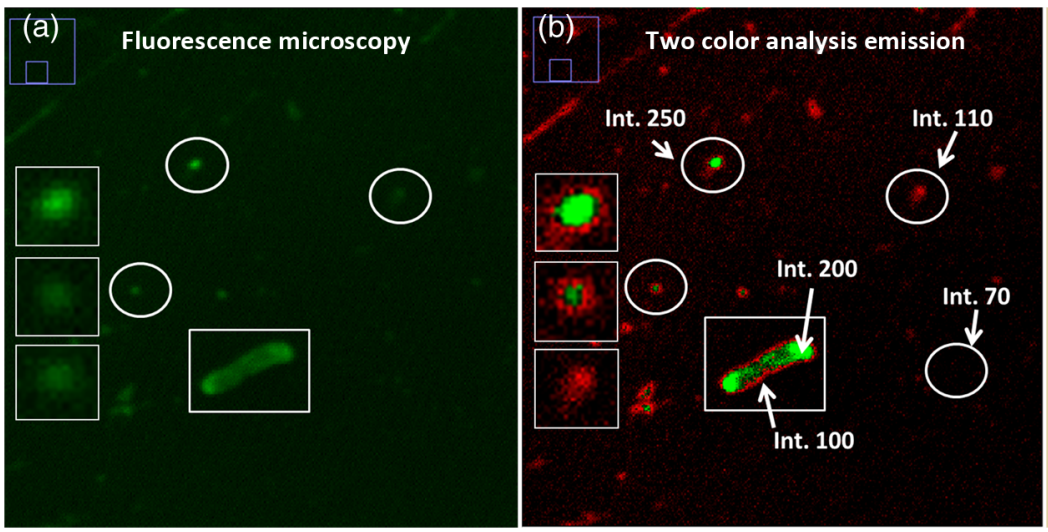

Fig. 10 (a) Fluorescence microscopy of E. coli bacteria labeled with luminescent gold core-shell nanoparticles [image edited with green color look-up-table (LUT)]. (b) Image edited by two-color analysis with red-green LUT. The insets are amplification of luminescent core-shell nanoparticles indicated with white circles (intensities $\times 10^{2}$ ).

By means of this nanoimaging editing, it was possible to obtain an improved detailed image resulting from the discrimination of different intensities collected as observed in Fig. 10(b).

\subsection{Effect of the Deposition of $\mathrm{AU} @ \mathrm{SiO}_{2}$ Nanoparticles on Luminescent Emission Over Bacteria}

To study the effect of AU@ $\mathrm{SiO}_{2}-\mathrm{RhB}$ nanoparticles' deposition, different concentrations of nanoparticles were added in the presence of an intermediate concentration of bacteria (see Fig. 9). In the absence of nanoparticles, no image was recorded, and a weak background signal was measured. Under diluted conditions, it was possible to detect micro-aggregates and individual bacteria with a weak acceptable signal; however, with a five-time increase in nanoparticle concentration, better images were obtained [see Figs. 11(a) and 11(b)]. As shown from the intensity analysis of the nanoimaging, two distributions of intensities were recorded [Fig. 11(c)]. It is important to mention that even under diluted conditions, it was possible to detect individual nanoparticles with a high-intensity emission signals [see circles surrounding nanoparticles, Fig. 11(a)]. 

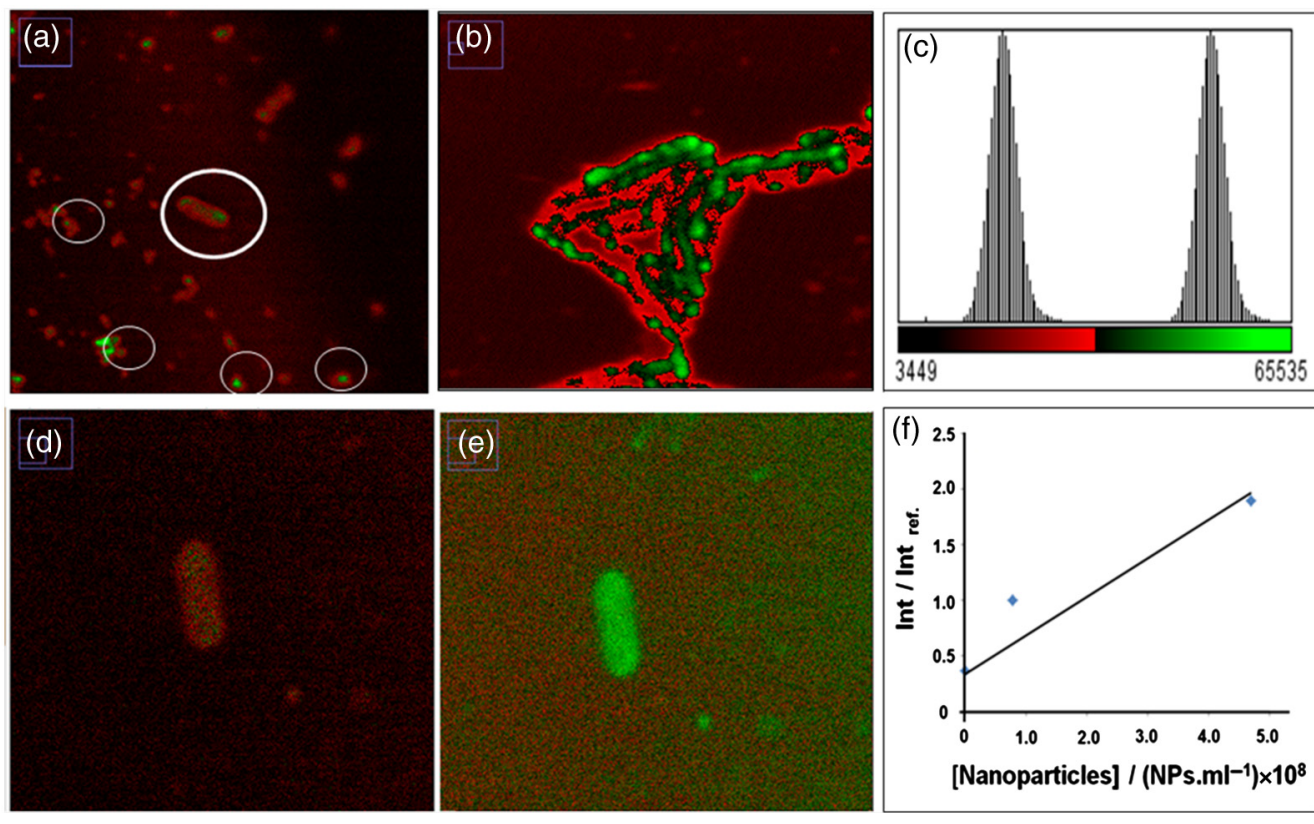

Fig. 11 (a) Fluorescence microscopy of $E$. coli bacteria labeled with diluted luminescent gold core-shell nanoparticles. (b) Fluorescence microscopy of $E$. coli bacteria labeled with luminescent gold core-shell nanoparticles under concentrated conditions. (c) Distribution of luminescent intensities from nanoimaging analysis (green distribution shows higher luminescence intensity than the red one). (d) Fluorescent microscopy of individual E. coli bacteria labeled from diluted bacteria concentration, with diluted luminescent gold core-shell nanoparticles. (e) Edited (d) image modifying the background color. (f) Calibration curve of bacterial detection by addition of different nanoparticle concentrations.

In the presence of diluted concentrations of nanoparticles, we could detect individual bacteria with the lower intensity distribution; in addition, improved images by editing the signal color background [see Figs. 11(d) and 11(e)] were obtained. The calibration curve for bacteria detection by addition of different nanoparticle concentrations showed a linear relationship [see Fig. 11(f)]. In this way, the importance of nanoparticle concentration addition for the detection based on nanoimaging analysis can be shown.

For biodetection, the following parameters should be considered to develop a new analytical methodology. The signal collected from the biological structure is particularly important to detect individual pathogens with high sensitivity, small volumes of real samples, and low limit of detections (LODs) are required. For fluorescent bacteria labeling, many approaches were developed as fluorescent silica nanoparticles, Qdots, fluorophores, fluorescent proteins with specific interaction with membranes, ${ }^{21}$ and synthetic fluorescent polymers with noncovalent interactions. ${ }^{22,23}$

For image resolution of bacterial fluorescent labeling, it is a very important parameter to take in account due to the information derived from the data collected.

As far as we know, no ultraluminescent gold core-shell nanoparticles for bacterial fluorescent labeling have been reported having the resolution and detail of bacterial imaging based on nanoimaging analysis. These nanostructures combine the biocompatible plasmonic properties of gold nanoparticles with the dielectric properties from the silica shell, a platform for bioconjugation with targeted applications. In a next step, we propose to use this approach in cytometry and application of microfluidic devices ${ }^{24,25}$ with detection based on nanoimaging. ${ }^{26}$

\section{Conclusions}

A nanoimaging platform was developed on the basis of ultraluminescent core-shell nanoparticles for detection of Escherichia coli bacteria. The thickness of the silica shell of the coreshell nanoparticles was improved, allowing the increase of the $\mathrm{MEF}_{\mathrm{EF}}$ to around 10. These 
luminescent nanoparticles were adsorbed over the bacteria, therefore, individual bacteria were detected under diluted and concentrated nanoparticle conditions. For bacteria detection, an effect of bacteria concentration was observed. At high and low concentrations of bacteria, a decreased emission was found, whereas at intermediate concentrations, a $40 \%$ increase was measured. Under these conditions, three distributions of ultraluminescent nanoparticles were measured: one with clear and strong green color (100\%), another with a strong green luminescent core surrounded by a weaker red emission, and another one with a weaker red color intensity (50\%).

In the absence of ultraluminescent nanoparticles, it was not possible to detect bacteria; however, by bright-field confocal microscopy, less-defined microstructures were recorded as compared with fluorescent labeled samples. Even for individual bacterial detection and diluted ultraluminescent core-shell nanoparticles concentrations, it was possible to detect bacteria based on nanoimaging.

For these reasons, with the nanoimaging platform developed, it will be possible to apply it to microfluidic systems, cytometry with fluorescent detection, and new technological approaches with nanoimaging detection of individual bacteria. Hence, it will be possible to develop a less time-consuming bioanalytical methodology, with minimal volumes required and a lower LOD.

\section{Acknowledgments}

We are greatly thankful for the different grants received to accomplish this work. We would also like to thank the Clinical and Bacteriological Analysis Laboratory (Laboratorio de Análisis Clínicos y Bacteriológicos, Clínica de la Familia II, Río Tercero, Argentina). In addition, we especially thank Professor Denis Boudreau from the Center for Optics, Photonics and Laser, Quebec, Canada (Département de chimie and Centre d'optique, photonique et lasers, Québec, Canada) for research work collaboration in progress. Finally, we thank and greatly appreciate the English revision done by Lucia V. Bracamonte.

\section{References}

1. O. Lazcka, F. J. Del Campo, and F. X. Muñoz, "Pathogen detection: a perspective of traditional methods and biosensors," Biosens. Electron. 22, 1205-1217 (2007).

2. H. J. Chung et al., "A magneto-DNA nanoparticle system for rapid detection and phenotyping of bacteria," Nat. Nanotechnol. 8, 369-375 (2013).

3. S. C. B. Gopinatha et al., "Bacterial detection: from microscope to smartphone," Biosens. Bioelectron. 60, 332-342 (2014).

4. L. Chen and J. Zhang, "Bioconjugated magnetic nanoparticles for rapid capture of grampositive bacteria," J. Biosens. Bioelectron. S11(5), 1-5 (2012).

5. J. Gao et al., "Combining fluorescent probes and biofunctional magnetic nanoparticles for rapid detection of bacteria in human blood," Adv. Mater. 18, 3145-3148 (2006).

6. J. Joo et al., "A facile and sensitive detection of pathogenic bacteria using magnetic nanoparticles and optical nanocrystal probes," Analyst 137(16), 3609-3612 (2012).

7. E. Arshad et al., "Fluorescence detection of the pathogenic bacteria Vibrio harveyi in solution and animal cells using semiconductor quantum dots," RSC Adv. 6, 15686-15693 (2016).

8. K.-S. Ahn et al., "Fluorescence energy transfer inhibition bioassay for cholera toxin based on galactose-stabilized gold nanoparticles and amine-terminated quantum dots," Microchem. J. 124, 9-14 (2016).

9. U. Resch-Genger et al., "Quantum dots versus organic dyes as fluorescent labels," Nat. Methods 5, 763-775 (2008).

10. J. R. Lackowicz, "Radiative decay engineering: metal enhanced fluorescence and plasmon emission," Anal. Biochem. 337, 171-194 (2005).

11. P. M. Fratamico et al., "Detection of Escherichia coli O157:H7 using a surface plasmon resonance biosensor," Biotechnol. Technol. 12(7), 571-576 (1998).

12. L. Zhang et al., "Multifunctional magnetic-plasmonic nanoparticles for fast concentration and sensitive detection of bacteria using SERS," Biosens. Bioelectron. 31, 130-136 (2012). 
13. T.-R. Teschner, L. Könözsy, and K. W. Jenkins, "Progress in particle-based multiscale and hybrid methods for flow applications," Microfluid Nanofluid 20(68), 1-38 (2016).

14. D. Boudreau et al., "Direct molecular detection of SRY gene from unamplified genomic DNA by metal-enhanced fluorescence and FRET," Anal. Methods 5, 6896-6899 (2013).

15. J. Asselin et al., "Correlating metal-enhanced fluorescence and structural properties in $\mathrm{Ag} @ \mathrm{SiO}_{2}$ core-shell nanoparticles," Plasmonics 11 1-8 (2016).

16. D. Gontero et al., "Synthesis of ultraluminiscent gold core-shell nanoparticles as nanoimaging platforms for biosensing applications based on metal enhanced fluorescence," RSC Adv. 7, 10252-10258 (2017).

17. J. Kimling et al., "Turkevich method for gold nanoparticle synthesis revisited," J. Phys. Chem. B 110, 15700-15707 (2006).

18. C. Graf et al., "A general method to coat colloidal particles with silica," Langmuir 19, 6693 6700 (2003).

19. D. Paramelle et al., "A rapid method to estimate the concentration of citrate capped silver nanoparticles from UV-visible light spectra," Analyst 139, 4855 (2014).

20. K. Aslan and C. D. Geddes, "Metal-enhanced chemiluminescence: advanced chemiluminescence concepts for the 21st century," Chem. Soc. Rev. 38, 2556-2564 (2009).

21. E. Gerard et al., "Fluorescence in situ hybridisation coupled to ultra-small immunogold detection to identify prokaryotic cells using transmission and scanning electron microscopy," J. Microbiol. Methods 63, 20-28 (2005).

22. M. D. Disney et al., "Detection of bacteria with carbohydrate-functionalized fluorescent polymers," J. Am. Chem. Soc. 126, 13343-13346 (2004).

23. J. Miao et al., "Imaging whole Escherichia coli bacteria by using single-particle x-ray diffraction," PNAS 100(1), 110-112 (2003).

24. M. Hayashi et al., "Fully automated on-chip imaging flow cytometry system with disposable contamination-free plastic re-cultivation chip," Int. J. Mol. Sci. 12, 3618-3634 (2011).

25. J. Skommer et al., "Multiparameter lab-on-a-chip flow cytometry of the cell cycle," Biosens. Bioelectron. 42(42), 586-591 (2013).

26. D. Brouard et al., "Nanoplasmonic sensing of nucleic acids using fluorescent core-shell nanoparticles and a cationic fluorescent polymer," ACS Nano 5, 1888-1896 (2011).

Daniela Gontero studied in the Faculty of Chemical Sciences, National University of Cordoba, Argentina, where she got the biochemist and pharmacy degrees in 2004 and 2007, respectively. After that she studied at Laval University, where she obtained her PhD in 2011. Now, she is completing the Biochemistry Specialist Program and holds a position at the Laboratory of Bacteriological and Clinical Analysis, Hospital of the Family II, Rio Tercero, Argentina.

Alicia V. Veglia holds a professor position at INFIQC, Institute of Physicochemical Research of Cordoba, Department of Organic Chemistry, National University of Cordoba (UNC), Argentina, and is a research group leader working on supramolecular chemistry, nanomaterials applied to nanosensors developments, analytical chemistry, and molecular modeling.

Denis Boudreau holds a professor position at Center of Laser, Optics, and Photonics (COPL) at Laval University, Québec, Canada, and he is a research group leader working on development of novel ultrasensitive analysis techniques based on laser spectroscopy or the latest advances in optics, nanoparticles, and biosensors for the purpose of studying trace atoms and molecules in various samples for environmental, industrial, or medical applications.

Angel Guillermo Bracamonte obtained his $\mathrm{PhD}$ at National University of Cordoba (UNC), Argentina. After that, he obtained a postdoctoral position at Laval University from 2009 to 2012 and University of Victoria Canada, from 2012 to 2013. He was research visitor at University of Regensburg, Germany, in 2013 and then held a research position at the University of Akron, USA, in 2014. Since 2015, he has held an assistant professor position at UNC and research position at CONICET (Commission of Research in Science, Argentina). 